\title{
Progress of graphene growth on copper by chemical vapor deposition: Growth behavior and controlled synthesis
}

\author{
MA LaiPeng ${ }^{1 *}$, REN WenCai $^{1 *}$, DONG ZaiLi $^{2}$, LIU LianQing $^{2}$ \& CHENG HuiMing ${ }^{1}$ \\ ${ }^{1}$ Shenyang National Laboratory for Materials Science, Institute of Metal Research, Chinese Academy of Sciences, Shenyang 110016, China; \\ ${ }^{2}$ State Key Laboratory of Robotics, Shenyang Institute of Automation, Chinese Academy of Sciences, Shenyang 110016, China
}

Received December 27, 2011; accepted March 16, 2012

\begin{abstract}
Recently, chemical vapor deposition (CVD) on copper has been becoming a main method for preparing large-area and highquality monolayer graphene. In this paper, we first briefly introduce the preliminary understanding of the microstructure and growth behavior of graphene on copper, and then focus on the recent progress on the quality improvement, number of layers control and transfer-free growth of graphene. In the end, we attempt to analyze the possible development of CVD growth of graphene in future, including the controlled growth of large-size single-crystal graphene and bilayer graphene with different stacking orders.
\end{abstract}

graphene, controlled growth, chemical vapor deposition, copper substrate

Citation: Ma L P, Ren W C, Dong Z L, et al. Progress of graphene growth on copper by chemical vapor deposition: Growth behavior and controlled synthesis. Chin Sci Bull, 2012, 57: 2995-2999, doi: 10.1007/s11434-012-5335-4

Graphene, a novel two-dimensional crystal, holds great promise in a wide range of applications such as electronics, transparent electrodes, energy storage, and functional composites due to its excellent properties such as giant carrier mobility, superior thermal conductivity, high transparency and good chemical stability [1,2]. Among all the developed synthesis methods, chemical vapor deposition (CVD) has been attracting increased interests for growing large-area highquality graphene film. In 2009, Li et al. [3] first realized the CVD growth of centimeter-sized graphene films dominated by monolayers (about $95 \%$ ) by using polycrystalline copper $\mathrm{Cu}$ foil as a substrate. These graphene films show high quality with a carrier mobility up to $4050 \mathrm{~cm}^{2} \mathrm{~V}^{-1} \mathrm{~s}^{-1}$. In contrast to the CVD growth of graphene on nickel (Ni), which follows a carbon segregation/precipitation mechanism, graphene grown on $\mathrm{Cu}$ is superior in both the controllability and uniformity of the number of layers due to the self-limiting surface adsorption growth mechanism [4]. Moreover, it has a great potential to grow very large films.

*Corresponding authors (email: lpma@imr.ac.cn; wcren@imr.ac.cn)
For example, Bae et al. [5] have realized the roll-to-toll production of 30 inch monolayer predominated graphene films. However, there is a large variation in the quality of graphene on $\mathrm{Cu}$ prepared by different groups with the carrier mobility varying from several hundreds to several thousands of $\mathrm{cm}^{2} \mathrm{~V}^{-1} \mathrm{~s}^{-1}$. These values are far below the theoretical limit $\left(\sim 10^{6} \mathrm{~cm}^{2} \mathrm{~V}^{-1} \mathrm{~s}^{-1}\right)$, and merely comparable to those of the commercial silicon materials. Meanwhile, it is necessary to develop the CVD process for growing highquality few- and multi-layer graphene since monolayer graphene is unable to satisfy the requirements of certain applications. In addition, it still remains a great challenge to realize the nondestructive and efficient transfer of large-area high-quality CVD grown graphene. Extensive efforts have been devoted to addressing the above issues in the past two years. In this paper, we will first briefly introduce the preliminary understanding of the microstructure and growth behavior of graphene on $\mathrm{Cu}$, and then focus on the recent progress on the quality improvement, number of layers control and transfer-free growth of graphene. In the end, we attempt to analyze the possible development of CVD growth 
of graphene in future.

\section{Preliminary understanding of the microstruc- ture and growth behavior of graphene on $\mathrm{Cu}$}

\subsection{Correlation between graphene growth and crystalline orientation of $\mathrm{Cu}$}

During the CVD growth process, $\mathrm{Cu}$ serves as both the growth substrate and the catalyst. Laminated $\mathrm{Cu}$ foils have been widely used for graphene growth, which are typical of polycrystalline structure and complex surface morphology. Therefore, it is necessary to clarify the influence of crystalline orientation and surface microstructure of $\mathrm{Cu}$ substrate on the structure and quality of graphene. Gao et al. [6] investigated the growth behavior of graphene on $\mathrm{Cu}(111)$ surface under ultra-high vacuum at $1000^{\circ} \mathrm{C}$, where ethylene was used as the carbon source. Scanning tunneling microscopy (STM) observations clearly revealed the heterogeneous nucleation of graphene on $\mathrm{Cu}(111)$ surface, in which graphene initially nucleates as sparse islands and gradually grows to "stitch" together to form larger domains. On the basis of moiré pattern between graphene and $\mathrm{Cu}$ substrate, there are two prominent alignments $\left(0^{\circ}\right.$ and $\left.7^{\circ}\right)$ of graphene islands over $\mathrm{Cu}(111)$ surface, while others are random alignments. Generally, no precise epitaxial alignment of graphene on $\mathrm{Cu}$ can be observed, consistent with the weak interactions between graphene and $\mathrm{Cu}$ substrate. In particular, a large amount of domain boundaries (or grain boundaries) were formed between the graphene domains (or grains) with various in-plane orientations, suggesting the polycrystalline nature of CVD graphene grown on $\mathrm{Cu}$ surface. However, strong correlation has been found between the surface crystalline orientation of $\mathrm{Cu}$ foil and the number of layers, quality and morphology of graphene. It seems that low-index $\mathrm{Cu}$ facets are more favorable for the formation of monolayer graphene with fewer defects as compared to the high-index surfaces [7]. Several independent studies have demonstrated the advantage of $\mathrm{Cu}(111)$ over other surfaces for growing high-quality monolayer graphene [7-9]. This has been ascribed to the different adsorption and diffusion processes of carbon-containing species over different $\mathrm{Cu}$ facets [7].

\subsection{Correlation between graphene growth and surface morphology of $\mathrm{Cu}$}

Early work has demonstrated that the monolayer graphene is continuous over $\mathrm{Cu}$ grain boundaries, implying that the surface morphology of $\mathrm{Cu}$ substrate has negligible effect on the continuity of graphene [3]. Detailed STM studies on the graphene grown on both polycrystalline $\mathrm{Cu}$ foil and singlecrystal $\mathrm{Cu}(100)$ indicated that the graphene layer remains atomically continuous while crossing the corrugated structures (e.g. atomic steps and vertices), surface defects (e.g. dislocations and atomic protrusions) $[10,11]$ and even amorphous regions on $\mathrm{Cu}$ [12]. These results present additional evidence to support that the interaction between graphene and $\mathrm{Cu}$ surface is weak. Yet, it was found that the conforming of graphene plane to the highly corrugated surface regions leads to the uneven strain and breaking of six-fold symmetry of the carbon lattice [12]. Orientation rotation was also observed while the graphene domains cross the step bunches of $\mathrm{Cu}$ surface, which inevitably leads to the formation of rotational boundaries within graphene domains [13]. In addition, the rough regions on $\mathrm{Cu}$ with more defects facilitate the nucleation of polycrystalline graphene while flat regions favor the formation of singlecrystal graphene [13]. Therefore, smooth $\mathrm{Cu}$ surface would be desirable for growing high-quality single-crystal graphene domains.

\subsection{Microstructure and grain boundaries of polycrys- talline graphene grown on $\mathrm{Cu}$}

Although Gao et al. [6] have shown the polycrystalline nature of graphene layer grown by CVD on $\mathrm{Cu}$, it still remains a challenge to characterize the microstructure of grain boundaries and to analyze the corresponding grain size distribution. Recently, the atomic structure of grain boundaries of CVD grown graphene has been revealed by researchers from Cornell University using aberration-corrected annular dark-field scanning transmission electron microscopy [14]. Their results show that graphene grains of different orientations are stitched together by a series of pentagons, heptagons and distorted hexagons. They also obtained the map of graphene grains by using dark-field transmission electron microscopy, in which the grain size can be directly measured. Comparison of the grain size distribution of graphene prepared by different CVD process suggested that the average grain size increases with increasing the purity of $\mathrm{Cu}$ substrate and the heating rate. Similar results have been observed by the researchers from University of California at Berkley and Lawrence Berkeley National Laboratory using the same characterization method [15]. Statistic analyses revealed the presence of preferential misorientations around $0^{\circ}$ and $30^{\circ}$, with others being randomly aligned. However, the statistic results of 46 grain boundaries reported by An et al. [16] showed that the misorientations of graphene grains are mainly located between $11^{\circ}$ and $30^{\circ}$. Such variations may be attributed to the differences of graphene microstructures obtained by different growth conditions.

\section{Controlled growth of graphene by CVD}

\subsection{Improvement of graphene quality}

On the basis of the studies of the correlation between graphene growth and crystalline orientation of $\mathrm{Cu}$ substrate, it would be an effective approach to grow high-quality monolayer graphene by using $\mathrm{Cu}(111)$ predominated $\mathrm{Cu}$ foil/film 
or single-crystal $\mathrm{Cu}(111)$ [7-9]. Reddy et al. [17] used single-crystal $\mathrm{Cu}(111)$ film thermally evaporated on $\alpha$ $\mathrm{Al}_{2} \mathrm{O}_{3}(0001)$ as a substrate to grow graphene at $1000^{\circ} \mathrm{C}$ by CVD. Raman measurements showed that monolayer predominated graphene films were formed on single-crystal $\mathrm{Cu}$ (111) surface with fewer defects compared to those grown on polycrystalline $\mathrm{Cu}$ substrates. $\mathrm{Hu}$ et al. [18] used similar substrate and compared the microstructure of graphene grown at 900 and $1000^{\circ} \mathrm{C}$. They also found that $\mathrm{Cu}(111)$ surface favors the formation of monolayer graphene, with superior uniformity to those grown on polycrystalline $\mathrm{Cu}$. Moreover, the growth temperature significantly influences the quality of graphene. The samples grown at $1000^{\circ} \mathrm{C}$ exhibited a high quality while those obtained at $900^{\circ} \mathrm{C}$ contained a large number of defects. Low energy electron diffraction analysis suggested the epitaxial growth of graphene on $\mathrm{Cu}(111)$ surface at $1000^{\circ} \mathrm{C}$ with the misorientations of $0^{\circ}$. In contrast, only a preferred alignment angle of $7^{\circ}$ was found for the samples grown at $900^{\circ} \mathrm{C}$. Therefore, high growth temperature is essentially important for growing high-quality graphene. Although graphene layer remains atomically continuous upon crossing structural corrugations and defects in $\mathrm{Cu}$ substrate, the corrugated surface structure facilitates the formation of defects and strain in graphene $[12,13]$. In addition, it promotes the generation of cracks and large wrinkles in graphene upon transferred to target substrate since the graphene layer duplicates the corrugated morphology of substrate [19]. Luo et al. [20] examined the effects of surface roughness of $\mathrm{Cu}$ foil on the quality of graphene. They used the electropolishing process to decrease the surface roughness of $\mathrm{Cu}$ foil by 10-30 times, and found that the carrier moblity of the graphene thus obtained can be improved from $50-200 \mathrm{~cm}^{2} \mathrm{~V}^{-1} \mathrm{~s}^{-1}$ to $400-600 \mathrm{~cm}^{2} \mathrm{~V}^{-1} \mathrm{~s}^{-1}$ by using the polished $\mathrm{Cu}$ as a substrate under identical growth conditions. Alternatively, Han et al. [21] used chemical mechanical polishing method to decrease the surface roughness of $\mathrm{Cu}$ foil. Their results revealed that the smooth $\mathrm{Cu}$ surface not only improves the quality of graphene (lower sheet resistance) but also reduces the nucleation density, which increases the uniformity of large-area graphene film. Therefore, smooth and high-purity $\mathrm{Cu}$ substrate is preferred for growing high-quality graphene.

As a typical line defect in polycrystalline graphene, grain boundary tend to increase the carrier scattering in graphene, thus decreasing the carrier mobility. Theoretical analysis by Yazyev and Louie [22] indicated that the grain boundaries of specific structure might lead to the formation of transport gaps for charge carriers. Yu et al. [23] prepared singlecrystal graphene with hexagonal shape by using ambient pressure CVD (APCVD). They found that the grain boundaries significantly increase the electrical resistance by comparing the electron transport within and between the graphene grains. Therefore, realization of effective control over the grain boundary would improve the carrier transport of graphene and the consistency of graphene-based devices. To this end, one of the promising approaches for mitigating the effects of grain boundary is to reduce the number of grain boundaries by preparing large-size graphene grain. From the perspective of growing graphene by CVD, it is necessary to reduce the nucleation density of graphene for the growth of large graphene grains. Li et al. [24,25] demonstrated that the CVD growth parameters strongly influence the density of graphene nuclei. Generally, the density of graphene nuclei decreases with increasing the temperature, lowering the partial pressure and flow rate of methane [24]. To overcome the concomitant decrease in growth rate, a two-step growth process was developed to grow large-size graphene grains, in which a low graphene nuclei density was obtained using high-temperature, low partial pressure and flow rate, followed by rapid growth via increasing the partial pressure and flow rate. However, only moderate improvement was obtained with grain size increasing from $\sim 6$ to $\sim 12 \mu \mathrm{m}$. Their further studies showed that nearly $500 \mu \mathrm{m}$ graphene grain can be formed on the inner side of a $\mathrm{Cu}$-foil enclosure using a much lower methane flow rate and partial pressure [25]. This approach provides a novel route for growing large graphene grain on $\mathrm{Cu}$ substrate, but the mechanism involved needs further investigations. Given that graphene nuclei is preferentially formed at the surface defects, it is possible to further improve the size and quality of graphene grain by using smooth low-defect $\mathrm{Cu}(111)$ as substrates.

Recent studies showed that the concentration of hydrogen also plays an important role in improving the quality of graphene during CVD. Hydrogen was widely used to eliminate the oxygen-containing impurities (e.g. $\mathrm{O}_{2}, \mathrm{H}_{2} \mathrm{O}$ ) in the CVD growth of graphene due to its reducing effect at elevated temperature. However, Gao et al. [26] found that a high hydrogen concentration degrades the quality of graphene in a APCVD process as a result of the increased defects (e.g. grain boudaries) and wrinkles. Similarly, Vlassiouk et al. [27] demonstrated the presence of a critical value of hydrogen concentration for graphene growth. No graphene nucleation was observed below this value, while high hydrogen concentration caused a great degradation in the quality of graphene. Based on these observations, it was proposed that hydrogen has "dual effects" on the growth of graphene: the hydrogen atoms dissociated by hydrogen molecules at elevated temperature act as both the co-catalyst for methane dissociation and the etchant for graphene edges and inner defects. Therefore, the quality and size of graphene grains might be further improved by optimizing the hydrogen concentration.

\subsection{Control of the number of layers}

As mentioned above, monolayer predominant graphene film can be selectively grown on $\mathrm{Cu}$ substrate by CVD [3]. However, high-quality few- and multi-layer graphene is also highly desired since monolayer graphene is unable to satisfy the requirements of certain applications. For instance, mon- 
olayer graphene suffers from a serious current leakage in electronic devices due to its intrinsic zero bandgap [2]. Therefore, it is of great significance to synthesize graphene with desirable bandgap for its potential applications in electronics. One of the possible solutions is to use AB stacking bilayer graphene, which allows a gate-controlled, continuously tunable bandgap [28]. In addition, few-layer graphene is expected to show much better electrical conductivity compared to monolayer graphene, and therefore can be used as high-performance transparent electrodes. Recent studies have demonstrated a close correlation between the CVD growth parameters and the number of layers of graphene obtained, which enables the formation of bilayer and few-layer graphene on $\mathrm{Cu}$ substrate. The key to growing bilayer and few-layer graphene is the high concentration of active carbon species. Yan et al. [29] developed a novel epitaxy CVD method to grow bilayer graphene, which involvs the use of $\mathrm{Cu}$ placed upstream as the catalyst for dissociating carbon source to epitaxially deposit the second layer on the existing monolayer graphene, with the highest coverage of $67 \%$. Selected area electron diffraction measurements confirmed the the $\mathrm{AB}$ stacking of the bilayer graphene obtained. Lee et al. [30] have demonstrated that large-area continuous bilayer graphene film can be formed by simply decreasing the cooling rate of CVD process. However, Raman spectra suggested that the bilayer graphene film obtained is typical of non-AB stacking structure. Alternatively, Luo et al. [31] showed that continuous bilayer graphene film can be formed by a combination of high concentration of carbon source and prolonged growth time. Raman results showed that strongly coupled or AB stacking bilayer structure predominates in this film, but the film still suffers from non-uniformity with the presence of tri-layer and monolayer areas. Cai et al. [32] demonstrated that high concentration of carbon source allows the formation of few-layer graphene film on $\mathrm{Cu}$ foil by significantly increasing the partial pressure of methane in low-pressure CVD process, which, however, suffers from high concentration of structural defects. Robertson et al. [33] showed that fewlayer graphene can also be formed using high concentration of carbon source via APCVD. Their results revealed that the formation of few-layer graphene film starts from the island-shaped few-layer nuclei, which then grow and stitch together to form continuous film. However, it still remains a great challenge to control the stacking structure and to improve the uniformity of CVD grown bilayer and few-layer graphene film.

\subsection{Transfer-free growth of graphene}

For various applications, graphene is required to place on specific substrates, most of which are non-metallic. Wetchemical route has been widely used to transfer graphene from the metal growth substrates onto target substrates. However, the impurities (e.g. residues of transfer media) and cracks/holes introduced in this transfer process would inevitably degrade the quality and uniformity of CVD graphene [19]. Furthermore, the transfer process not only increases the cost but also leads to a low efficiency in the preparation of large-area graphene. In addition to developing novel transfer methods, transfer-free growth provides an alternative strategy towards solving this issue. Ismach et al. [34] used $\mathrm{Cu}$ film (450 $\mathrm{nm}$ in thickness) on Si wafer as a substrate to grow graphene at $1000^{\circ} \mathrm{C}$ and $13-65 \mathrm{~Pa}$. Upon the formation of graphene, $\mathrm{Cu}$ was evaporated by prolonging the heating time (up to $7 \mathrm{~h}$ ) at elevated temperature and low pressure, thus leaving the graphene in direct contact with the Si wafer. Although this approach precludes the impurities and possible damage involved in the wet chemical transfer, large amounts of defects are formed in graphene during this process, together with a low efficiency. Similar $\mathrm{Cu}$ film on $\mathrm{Si}$ wafer was used as a substrate for CVD growth of graphene by Su et al. [35]. They found that methane molecule would diffuse across the grain boundaries of $\mathrm{Cu}$ film and form graphene at the interfaces between $\mathrm{Cu}$ film and $\mathrm{Si}$ wafer upon decreasing the thickness of $\mathrm{Cu}$ film to $300 \mathrm{~nm}$ and lowering the growth temperature to $900^{\circ} \mathrm{C}$. Continuous graphene film could then be obtained on the surface of $\mathrm{Si}$ wafer by etching the $\mathrm{Cu}$ film with chemical etchant. In sharp contrast to a typical CVD process, it is difficult to directly control the concentration of carbon sources in the form of gas at the interfaces for this method. As a result, non-uniform few-layer graphene was obtained with a carrier mobility of $672 \mathrm{~cm}^{2} \mathrm{~V}^{-1} \mathrm{~s}^{-1}$. Recent progress in transfer-free growth of graphene on Ni substrate [36] and CVD growth of graphene on $\mathrm{Cu}$ using solid carbon sources [37] suggests that the quality and uniformity of graphene formed at the interface might be further improved by using solid carbon sources.

\section{Concluding remarks}

Since the first report of CVD growth of large-area and highquality graphene on $\mathrm{Cu}$ foil in 2009 [3], CVD has attracted extensive attentions as an effective approach for synthesizing uniform monolayer graphene. The rapid development of CVD growth of graphene has strongly promoted the application explorations of graphene in a wide range of fields such as transparent conductive electrodes and radio frequency devices. However, it still remains a great challenge to improve the control over the quality and microstructure of graphene obtained by CVD. Recent studies have shown that structural defects of graphene can be reduced by controlling the surface crystalline orientation, lowering the surface roughness and reducing the surface defects of $\mathrm{Cu}$ substrate. Bilayer and few-layer graphene can be formed by modifying the CVD process and optimizing the growth parameters. Interfacial growth between metal film and nonmetal substrate enables the direct formation of relatively 
high-quality graphene on the non-metal substrates (e.g. Si wafer). Realization of effective control over the grain boundary, which is the typical line defect in polycrystalline graphene, would increase the carrier transport of graphene and the consistency of graphene-based devices. Thus, it is highly desirable to grow large-size high-quality singlecrystal graphene by using CVD in future. In addition, the application studies based on high-quality CVD graphene would be significantly facilitated by using uniform largearea high-quality bilayer and few-layer graphene combined with improved structural controllability. In particular, the control over the stacking of bilayer and few-layer graphene is crucially important, which largely depends on the understanding of nucleation and growth mechanism of graphene in the CVD process.

This work was supported by the National Natural Science Foundation of China (51102241) and State Key Laboratory of Robotics (RLO201012).

1 Geim A K, Novoselov K S. The rise of graphene. Nat Mater, 2007, 6: 183-191

2 Geim A K. Graphene: Status and prospects. Science, 2009, 324: 1530-1534

3 Li X S, Cai W W, An J H, et al. Large-area synthesis of high-quality and uniform graphene films on copper foils. Science, 2009, 324: 1312-1314

4 Li X S, Cai W W, Colombo L, et al. Evolution of graphene growth on $\mathrm{Ni}$ and $\mathrm{Cu}$ by carbon isotope labeling. Nano Lett, 2009, 9: 4268-4272

5 Bae S, Kim H, Lee Y, et al. Roll-to-roll production of 30-inch graphene films for transparent electrodes. Nat Nanotechnol, 2010, 5: 574-578

6 Gao L, Guest J R, Guisinger N P. Epitaxial graphene on $\mathrm{Cu}(111)$. Nano Lett, 2010, 10: 3512-3516

7 Wood J D, Schmucker S W, Lyons A S, et al. Effects of polycrystalline $\mathrm{Cu}$ substrate on graphene growth by chemical vapor deposition. Nano Lett, 2011, 11: 4547-4554

8 Ishihara M, Koga Y, Kim J, et al. Direct evidence of advantage of $\mathrm{Cu}(111)$ for graphene synthesis by using Raman mapping and electron backscatter diffraction. Mater Lett, 2011, 65: 2864-2867

9 Zhao L, Rim K T, Zhou H, et al. Influence of copper crystal surface on the CVD growth of large area monolayer graphene. Solid State Commun, 2011, 151: 509-513

10 Rasool H I, Song E B, Allen M J, et al. Continuity of graphene on polycrystalline copper. Nano Lett, 2011, 11: 251-256

11 Rasool H I, Song E B, Mecklenburg M, et al. Atomic-scale characterization of graphene grown on copper(100) single crystals. J Am Chem Soc, 2011, 133: 12536-12543

12 Zhang Y F, Gao T, Gao Y B, et al. Defect-like structures of graphene on copper foils for strain relief investigated by high-resolution scanning tunneling microscopy. ACS Nano, 2011, 5: 4014-4022

13 Nie S, Wofford J M, Bartelt N C, et al. Origin of the mosaicity in graphene grown on $\mathrm{Cu}(111)$. Phys Rev B, 2011, 84: 155425

14 Huang P Y, Ruiz-Vargas C S, van der Zande A M, et al. Grains and grain boundaries in single-layer graphene atomic patchwork quilts. Nature, 2011, 469: 389-393

15 Kim K, Lee Z, Regan W, et al. Grain Boundary mapping in polycrystalline graphene. ACS Nano, 2011, 5: 2142-2146

16 An J H, Voelkl E, Suk J W, et al. Domain (Grain) boundaries and evidence of "twinlike" structures in chemically vapor deposited grown graphene. ACS Nano, 2011, 5: 2433-2439

17 Reddy K M, Gledhill A D, Chen C H, et al. High quality, transferrable graphene grown on single crystal $\mathrm{Cu}(111)$ thin films on basal-plane sapphire. Appl Phys Lett, 2011, 98: 113117

$18 \mathrm{Hu} \mathrm{B}$, Ago H, Ito Y, et al. Epitaxial growth of large-area single-layer graphene over $\mathrm{Cu}(111) /$ sapphire by atmospheric pressure CVD. Carbon, 2012, 50: 57-65

19 Li X S, Zhu Y W, Cai W W, et al. Transfer of large-area graphene films for high-performance transparent conductive electrodes. Nano Lett, 2009, 9: 4359-4363

20 Luo Z T, Lu Y, Singer D W, et al. Effect of substrate roughness and feedstock concentration on growth of wafer-scale graphene at atmospheric pressure. Chem Mater, 2011, 23: 1441-1447

21 Han G H, Gunes F, Bae J J, et al. Influence of copper morphology in forming nucleation seeds for graphene growth. Nano Lett, 2011, 11: 4144-4148

22 Yazyev O V, Louie S G. Electronic transport in polycrystalline graphene. Nat Mater, 2010, 9: 806-809

23 Yu Q K, Jauregui L A, Wu W, et al. Control and characterization of individual grains and grain boundaries in graphene grown by chemical vapour deposition. Nat Mater, 2011, 10: 443-449

24 Li X S, Magnuson C W, Venugopal A, et al. Graphene films with large domain size by a two-step chemical vapor deposition process. Nano Lett, 2010, 10: 4328-4334

25 Li X S, Magnuson C W, Venugopal A, et al. Large-area graphene single crystals grown by low-pressure chemical vapor deposition of methane on copper. J Am Chem Soc, 2011, 133: 2816-2819

26 Gao L B, Ren W C, Zhao J P, et al. Efficient growth of high-quality graphene films on $\mathrm{Cu}$ foils by ambient pressure chemical vapor deposition. Appl Phys Lett, 2010, 97: 183109

27 Vlassiouk I, Regmi M, Fulvio P, et al. Role of hydrogen in chemical vapor deposition growth of large single-crystal graphene. ACS Nano, 2011, 5: 6069-6076

28 Zhang Y B, Tang T T, Girit C, et al. Direct observation of a widely tunable bandgap in bilayer graphene. Nature, 2009, 459: 820-823

29 Yan K, Peng H L, Zhou Y, et al. Formation of bilayer bernal graphene: Layer-by-layer epitaxy via chemical vapor deposition. Nano Lett, 2011, 11: 1106-1110

30 Lee S, Lee K, Zhong Z H. Wafer scale homogeneous bilayer graphene films by chemical vapor deposition. Nano Lett, 2010, 10: 4702-4707

31 Luo Z Q, Yu T, Shang J Z, et al. Large-scale synthesis of bi-layer graphene in strongly coupled stacking order. Adv Funct Mater, 2011, 21: 911-917

32 Cai W W, Zhu Y W, Li X S, et al. Large area few-layer graphene/ graphite films as transparent thin conducting electrodes. Appl Phys Lett, 2009, 95: 123115

33 Robertson A W, Warner J H. Hexagonal single crystal domains of few-layer graphene on copper foils. Nano Lett, 2011, 11: 1182-1189

34 Ismach A, Druzgalski C, Penwell S, et al. Direct chemical vapor deposition of graphene on dielectric surfaces. Nano Lett, 2010, 10: $1542-1548$

35 Su C Y, Lu A Y, Wu C Y, et al. Direct formation of wafer scale graphene thin layers on insulating substrates by chemical vapor deposition. Nano Lett, 2011, 11: 3612-3616

36 Shin H, Choi W M, Yoon S M, et al. Transfer-free growth of fewlayer graphene by self-assembled monolayers. Adv Mater, 2011, 23: 4392-4397

37 Sun Z Z, Yan Z, Yao J, et al. Growth of graphene from solid carbon sources. Nature, 2010, 468: 549-552

Open Access This article is distributed under the terms of the Creative Commons Attribution License which permits any use, distribution, and reproduction in any medium, provided the original author(s) and source are credited. 